\title{
Inverse Thermoelastic Analysis of a Thick Rectangular Plate
}

\author{
Sanjay H Bagade
}

\begin{abstract}
Thermal stresses and displacement functions are obtained for a rectangular plate occupying the space $R:-a \leq x \leq$ $a, 0 \leq y \leq b,-h \leq z \leq h$, with the known boundary and initial conditions. In this inverse problem the unknown surface temperature is determined on the boundary along the y-axis when the temperature at some internal point is known. The governing heat conduction equation has been solved by applying Marchi - Fasulo transform and Laplace transform techniques. The solutions are obtained in form of infinite series. The results for displacement and thermal stresses have been computed numerically and illustrated graphically for Aluminium plate. MSC 2010: 74A10,74J25, 74H99, 74D99
\end{abstract}

Keywords: Rectangular plate, Inverse thermoelastic problem, Integral transform, Thermal stress.

\section{INTRODUCTION}

Thermal stresses in material are one of the important factors which affect the life of the material bodies. Thermal stresses occurring in the body due to heat distribution, may often lead to breakdown and failures in engineering structures. This necessitates a deep thermal and mechanical analysis of a material structure before it is put to engineering application. Different mathematical models and methodologies are employed for thermoelastic studies, and few are enlisted here.Temperature distribution and resultant stress were derived for a rectangular slab on the basis of the general theory of thermoelasticity (Grysa \& Kozłowski, 1983; Xue et al., 2019; Youssef et al., 2019).The LanczosChebyshev method was applied to study the fluctuating temperatures and thermal stresses in a thin rectangular plate subjected to heat losses, and results obtained were in power series (Chen, 1988). Thermal response in a solid structure made up of composite material and subjected to thermal shock was discussed by using heat conduction equations for orthotropic material (Robert J. Adams, 1999). Exact solutions for a functionally graded cylindrical structures, with general boundary conditions, were obtained to analyze the steady state as well unsteady state heat conduction in them (Amiri Delouei, Emamian, Karimnejad, \& Sajjadi, 2019; Amiri Delouei, Emamian, Karimnejad, Sajjadi, et al., 2019; Delouei et al., 2020).

Manuscript received on July 07, 2021.

Revised Manuscript received on July 12, 2021.

Manuscript published on July 30, 2021.

* Correspondence Author

Sanjay H Bagade*, Department of Physics, Janki Devi Bajaj College of Science, Wardha (Maharashtra), India. E-mail: sanjaybagade8@gmail.com

(C) The Authors. Published by Blue Eyes Intelligence Engineering and Sciences Publication (BEIESP). This is an open access article under the CC BY-NC-ND license (http://creativecommons.org/licenses/by-nc-nd/4.0/)

Time varying thermal stresses and temperature distribution in a three-dimensional FGM rectangular plate were determined by using the theory of laminated composites (M. Marin \& Craciun, 2017; Marin Marin \& Öchsner, 2017; Noda et al., 1989; Tanigawa, Yoshihiro Ootao, 1999). in these solids (Arslantürk, 2018; Güngör \& Arslantürk, 2019). Lata and Kaur used the Laplace and Fourier transform techniques as well as the potential function method to determine the temperature, displacement, and stresses in isotropic circular solids (I. Kaur \& Lata, 2019; Lata. P. and Kaur, 2019). The finite Fourier sine transform technique was used to deal with inverse steady state problem and obtain the strain-stress at the boundary of a thin rectangular plate (Manthena, V., Lamba, N., Kedar, 2018). Thermal response was studied by use of integral transform technique and Kirchhoff's variable transformation in solid bodies subjected to heat source having hyperbolic variation (Ahire et al., 2020). The heat conduction theory which makes use of differential transforms was employed in derivation of thermal stress in rectangular plate (Chaudhari \& Sutar, 2019). Roy et al. computed thermal stresses in a semi-infinite rectangular beam by application of sine transform methods (Roy H., 2013). Thus, literature survey points towards use of different models and methods for studying the thermoelastic profile of different solid structures.

In the present article, an inverse problem is studied to determine the displacement and thermal stresses in rectangular plate occupying the region $\mathrm{R}:-\mathrm{a} \leq \mathrm{x} \leq \mathrm{a}, 0 \leq \mathrm{y} \leq$ $\mathrm{b},-\mathrm{h} \leq \mathrm{z}<\mathrm{h}$ with the known boundary and initial conditions. The unknown surface temperature is determined on the boundary along the y-axis, when the temperature at some internal point is known and both the boundaries along $\mathrm{x}$ and $\mathrm{z}$-axis are maintained at zero temperature. The governing heat conduction equation have been solved by applying Marchi - Fasulo transform and Laplace transform techniques. The solutions are obtained in form of infinite series. The results for displacement and thermal stresses have been computed numerically and illustrated graphically for a thick rectangular plate of aluminium metal.

\section{MATHEMATICAL FORMULATIONS}

A rectangular plate occupying the region $\mathrm{R}$ : $-\mathrm{a} \leq \mathrm{x} \leq \mathrm{a}, 0$ $\leq \mathrm{y} \leq \mathrm{b},-\mathrm{h} \leq \mathrm{z} \leq \mathrm{h}$ is considered. The displacement components $u_{x}, u_{y}$ and $u_{z}$ along the $X, Y, Z$ direction respectively are given in the integral form as in (Noda et al., 2003)

$u_{x}=\int_{-a}^{a} \frac{1}{E}\left(\frac{\partial^{2} u}{\partial y^{2}}+\frac{\partial^{2} u}{\partial z^{2}}-v \frac{\partial^{2} u}{\partial x^{2}}+\alpha T\right) d x$

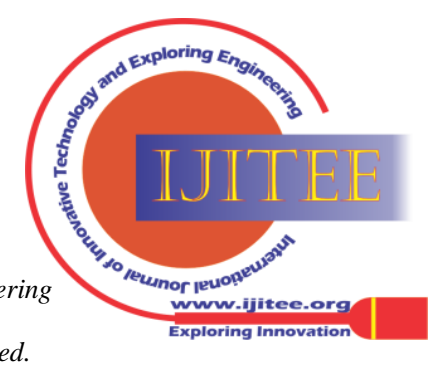


$u_{y}=\int_{0}^{b} \frac{1}{E}\left(\frac{\partial^{2} u}{\partial z^{2}}+\frac{\partial^{2} u}{\partial x^{2}}-v \frac{\partial^{2} u}{\partial y^{2}}+\alpha T\right) d y$

$u_{z}=\int_{-h}^{h} \frac{1}{E}\left(\frac{\partial^{2} u}{\partial x^{2}}+\frac{\partial^{2} u}{\partial y^{2}}-v \frac{\partial^{2} u}{\partial z^{2}}+\alpha T\right) d z$

Where $E, v$ and $\alpha$ are the Young's modulus, poisons ratio and the linear coefficient of thermal expansion of the material of the plate respectively and $\cup(x, y, z, t)$ is the Airy's stress function which satisfy the differential equation

$\left(\frac{\partial^{2}}{\partial x^{2}}+\frac{\partial^{2}}{\partial y^{2}}+\frac{\partial^{2}}{\partial z^{2}}\right)^{2} \cup(x, y, z, t)=-\alpha E\left(\frac{\partial^{2}}{\partial x^{2}}+\frac{\partial^{2}}{\partial y^{2}}+\right.$

$\left.\frac{\partial^{2}}{\partial z^{2}}\right) \quad T(x, y, z, t)$

Where $T(x, y, z, t)$ denotes the temperature of thin rectangular plate satisfying the following differential equation [21]

$\frac{\partial^{2} T}{\partial x^{2}}+\frac{\partial^{2} T}{\partial y^{2}}+\frac{\partial^{2} T}{\partial z^{2}}=\frac{1}{k} \frac{\partial T}{\partial t}$

(5)

here $k$ is thermal diffusivity of the material. The initial condition is

$T(x, y, z, 0)=0$

and the boundary conditions are

$\left[T(x, y, z, t)+k_{1} \frac{\partial T(x, y, z, t)}{\partial x}\right]_{x=a}=0$

$\left[T(x, y, z, t)+k_{2} \frac{\partial T(x, y, z, t)}{\partial x}\right]_{x=-a}=0$

$[T(x, y, z, t)]_{y=b}=G(x, z, t)$

(Unknown)

(9)

$\left[T(x, y, z, t)+C \frac{\partial T(x, y, z, t)}{\partial y}\right]_{y=0}=g(x, z, t)$

$\left[T(x, y, z, t)+k_{3} \frac{\partial T(x, y, z, t)}{\partial z}\right]_{z=h}=0$

$\left[T(x, y, z, t)+k_{4} \frac{\partial T(x, y, z, t)}{\partial z}\right]_{z=-h}=0$

The interior condition is

$\left[T(x, y, z, t)+c \frac{\partial T(x, y, z, t)}{\partial z}\right]_{y=\xi}=f(x, z, t)$

(Known)

The stresses components in terms of $U(x, y, z, t)$ are given by

$$
\begin{aligned}
& \sigma_{x x}=\left(\frac{\partial^{2} u}{\partial y^{2}}+\frac{\partial^{2} u}{\partial z^{2}}\right) \\
& \sigma_{y y}=\left(\frac{\partial^{2} u}{\partial z^{2}}+\frac{\partial^{2} u}{\partial x^{2}}\right) \\
& \sigma_{z z}=\left(\frac{\partial^{2} u}{\partial x^{2}}+\frac{\partial^{2} u}{\partial y^{2}}\right)
\end{aligned}
$$

(16)

The equations (1) to (16) constitute the mathematical formulation of the problem under consideration.

\section{MATHEMATICAL SOLUTIONS}

By applying finite Marchi - Fasulo transform and Laplace transform to the equations (5) to (13), and then taking their inversion, we obtain

$$
\begin{aligned}
& T(x, y, z, t)=\frac{k}{c^{2}} \sum_{m, n=1}^{\infty}\left[\frac{P_{m}(x)}{\lambda_{m}}\right]\left[\frac{P_{n}(z)}{\lambda_{n}}\right]\left[\varphi_{1}(y) \tau_{1}(t)-\right. \\
& \left.\varphi_{2}(y) \tau_{2}(t)\right] \\
& +\frac{2 k \pi}{\xi^{2}} \sum_{m, n, \zeta=1}^{\infty}\left[\frac{P_{m}(x)}{\lambda_{m}}\right]\left[\frac{P_{n}(z)}{\lambda_{n}}\right]\left[\frac{\varsigma}{\cos \varsigma \pi}\right]\left[\frac{\psi_{1}(y) \tau_{3}(t)-\psi_{2}(y) \tau_{4}(t)}{\left[1+(c \varsigma \pi / \xi)^{2}\right]}\right] \\
& -\sum_{m, n=1}^{\infty}\left[\frac{P_{m}(x)}{\lambda_{m}}\right]\left[\frac{P_{n}(z)}{\lambda_{n}}\right] A_{3}(m, n, y, t) \\
& -\sum_{m, n=1}^{\infty}\left[\frac{P_{m}(x)}{\lambda_{m}}\right]\left[\frac{P_{n}(z)}{\lambda_{n}}\right] A_{3}(m, n, b, t) \\
& \varphi_{1}(y)=\frac{\sinh \left(\frac{y}{c}\right)-\cosh \left(\frac{y}{c}\right)}{\sinh (\xi / c)}, \varphi_{2}(y)=
\end{aligned}
$$

$\frac{\sinh \left(\frac{y-\xi}{c}\right)-\cosh \left(\frac{y-\xi}{c}\right)}{\sinh (\xi / c)}$

$$
\begin{aligned}
& \psi_{1}(y)= \sin \left(\frac{\varsigma \pi}{\xi}\right) y-\left(\frac{c \varsigma \pi}{\xi}\right) \cos \left(\frac{\varsigma \pi}{\xi}\right) y \\
& \psi_{2}(y)=\sin \left(\frac{\varsigma \pi}{\xi}\right)(y-\xi) \\
&-\left(\frac{c \varsigma \pi}{\xi}\right) \cos \left(\frac{\varsigma \pi}{\xi}\right)(y
\end{aligned}
$$$$
-\xi)
$$

$$
\begin{aligned}
\tau_{1}(t)=\int_{0}^{t}[\overline{\bar{f}}(m, n, t-u) & \\
& -A_{1}(m, n, t \\
& -u)] e^{k u\left[\frac{1-c^{2} q^{2}}{c^{2}}\right]} d u
\end{aligned}
$$

$\tau_{2}(t)=\int_{0}^{t} \overline{\bar{g}}(m, n, t-u)-A_{2}(m, n, t$

$$
-u) e^{k u\left[\frac{1-c^{2} q^{2}}{c^{2}}\right]} d u
$$

$$
\begin{array}{r}
\tau_{3}(t)=\int_{0}^{t}\left[\overline{\bar{f}}(m, n, t-u)-A_{1}(m, n, t\right. \\
-u)] e^{-k u\left[q^{2}+\left(\frac{\varsigma \pi}{\xi}\right)^{2}\right]} d u \\
\tau_{4}(t)=\int_{0}^{t}\left[\overline{\bar{g}}(m, n, t-u)-A_{2}(m, n, t\right. \\
-u) e^{-k u\left[q^{2}+\left(\frac{\left(\pi^{2}\right.}{\xi}\right)^{2}\right]} d u
\end{array}
$$

$A_{1}(m, n, t)=\left[\left(\chi+c \frac{d \chi}{d z}\right)_{z=\xi}\right], \quad A_{2}(m, n, t)=$

$\left[\left(\chi+c \frac{d \chi}{d z}\right)_{z=0}\right]$

Published By:

Blue Eyes Intelligence Engineering

Retrieval Number: 100.1/ijitee.I93230710921

DOI: 10.35940/ijitee.I9323.0710921

Journal Website: www.ijitee.org 
$A_{3}(m, n, z, t)=L^{-1}[\chi]$

Here $\overline{\bar{f}}(m, n, t)$ and $\overline{\bar{g}}(m, n, t)$ denote the Marchi Fasulo transforms of $\bar{f}(m, z, t)$ and $\bar{g}(m, z, t)$ respectively. $\bar{f}(m, z, t)$ and $\bar{g}(m, z, t)$ denote the finite Marchi - Fasulo transform of $f(x, z, t)$ and $g(x, z, t)$ respectively.

$\bar{f}(m, n, t)=\int_{-h}^{h} \bar{f}(m, z, t) P_{n}(z) d z, \quad \bar{g}(m, n, t)=$ $\int_{-h}^{h} \bar{g}(m, z, t) P_{n}(z) d z, \quad \quad \lambda_{n}=\int_{-h}^{h} P_{n}^{2}(z) d z$

$P_{n}(z)=Q_{n} \cos \left(a_{n} z\right)-W_{n} \sin \left(a_{n} z\right)$

$Q_{n}=a_{n}\left(\alpha_{3}+\alpha_{4}\right) \cos \left(a_{n} h\right)+\left(\beta_{3}-\beta_{4}\right) \sin \left(a_{n} h\right)$

$W_{n}=\left(\beta_{3}+\beta_{4}\right) \cos \left(a_{n} h\right)+\left(\alpha_{4}-\alpha_{3}\right) a_{n} \sin \left(a_{n} h\right)$

Equation (17) is the desired solution of the given problem with $\beta_{3}=\beta_{4}=1$,

$\alpha_{3}=k_{3}, \quad \alpha_{4}=k_{4}$.

\subsection{Determination Of Airys Stress Function:}

Substituting the values of $T(x, y, z, t)$ from equation (17) in equation (4) one obtains $U(x, y, z, t)=$ $\frac{\alpha E k}{c^{2}} \sum_{m, n=1}^{\infty}\left[\frac{P_{m}(x)}{\lambda_{m}}\right]\left[\frac{P_{n}(z)}{\lambda_{n}}\right]\left[\frac{\left[\varphi_{1}(z) \tau_{1}(t)-\varphi_{2}(z) \tau_{2}(t)\right]}{a_{m}^{2}+a_{n}^{2}-1 / c^{2}}\right]$

$+\frac{2 \alpha E k \pi}{\xi^{2}} \sum_{m, n, \zeta=1}^{\infty}\left[\frac{P_{m}(x)}{\lambda_{m}}\right]\left[\frac{P_{n}(z)}{\lambda_{n}}\right]\left[\frac{\varsigma}{\cos \varsigma \pi}\right]\left[\frac{1}{1+(\operatorname{c\varsigma } \pi / \xi)^{2}}\right]$ $\times\left[\frac{\psi_{1}(z) \tau_{3}(t)-\psi_{2}(z) \tau_{4}(t)}{a_{m}^{2}+a_{n}^{2}+(\varsigma \pi / \xi)^{2}}\right]$

$-\alpha E \sum_{m, n=1}^{\infty}\left[\frac{P_{m}(x)}{\lambda_{m}}\right]\left[\frac{P_{n}(z)}{\lambda_{n}}\right]\left[\frac{A_{3}(m, n, z, t)}{a_{m}^{2}+a_{n}^{2}-l_{0}}\right]$

\subsection{Determination of Displacement Components}

Substituting the values of (19) in the equation (1) to (3) one obtains

$$
\begin{aligned}
u_{x}=\frac{\alpha k}{c^{2}} \sum_{m, n=1}^{\infty}\left[\frac{\left(k_{1}+k_{2}\right) \sin 2 a_{m} a}{\lambda_{m}}\right]\left[\frac{P_{n}(z)}{\lambda_{n}}\right]\left[\frac{(1+v) a_{m}^{2}}{a_{m}^{2}+a_{n}^{2}-1 / c^{2}}\right] \\
\times\left[\varphi_{1}(y) \tau_{1}(t)-\varphi_{2}(y) \tau_{2}(t)\right] \\
+\frac{2 \alpha k \pi}{\xi^{2}} \sum_{m, n, \varsigma=1}^{\infty}\left[\frac{\left(k_{1}+k_{2}\right) \sin 2 a_{m} a}{\lambda_{m}}\right]\left[\frac{\varsigma}{\cos \varsigma \pi}\right]\left[\frac{P_{n}(z)}{\lambda_{n}}\right]\left[\frac{(1+v) a_{m}^{2}}{a_{m}^{2}+a_{n}^{2}+(\varsigma \pi / \xi)^{2}}\right] \\
\times\left[\frac{\psi_{1}(y) \tau_{3}(t)-\psi_{2}(y) \tau_{4}(t)}{1+(c \varsigma \pi / \xi)^{2}}\right]
\end{aligned}
$$

$-\alpha \sum_{m, n=1}^{\infty}\left[\frac{\left(k_{1}+k_{2}\right) \sin 2 a_{m} a}{\lambda_{m}}\right]\left[\frac{P_{n}(z)}{\lambda_{n}}\right]\left[\frac{(1+v) a_{m}^{2}}{a_{m}^{2}+a_{n}^{2}-l_{0}}\right] A_{3}(m, n, y, t)$

(20)

$$
\begin{aligned}
& u_{y}= \\
& \frac{\alpha k}{c^{2}} \sum_{m, n=1}^{\infty}\left[\frac{P_{m}(x)}{\lambda_{m}}\right]\left[\frac{P_{n}(z)}{\lambda_{n}}\right]\left[\frac{-(1+v) / c^{2}}{a_{m}^{2}+a_{n}^{2}-1 / c^{2}}\right]\left[\varphi_{1}^{\prime}(b) \tau_{1}(t)-\right. \\
& \begin{aligned}
& \varphi_{2}^{\prime}(b)\left.\tau_{2}(t)\right] \\
& \quad+\frac{2 \alpha k \pi}{\xi^{2}} \sum_{m, n, \zeta=1}^{\infty}\left[\frac{P_{m}(x)}{\lambda_{m}}\right]\left[\frac{P_{n}(z)}{\lambda_{n}}\right]\left[\frac{\varsigma}{\cos (\varsigma \pi)}\right] \\
&\left.\quad \frac{(1+v)(\varsigma \pi / \xi)^{2}}{a_{m}^{2}+a_{n}^{2}+(\varsigma \pi / \xi)^{2}}\right]\left[\frac{1}{\left(1+(c \varsigma \pi / \xi)^{2}\right.}\right] \\
& \quad \times\left[\psi_{1}^{\prime}(b) \tau_{3}(t)-\psi_{2}^{\prime}(b) \tau_{4}(t)\right]
\end{aligned}
\end{aligned}
$$

$$
\begin{gathered}
-\alpha \sum_{m, n=1}^{\infty}\left[\frac{P_{m}(x)}{\lambda_{m}}\right]\left[\frac{P_{n}(z)}{\lambda_{n}}\right]\left[\frac{-(1+v) l_{0}}{a_{m}^{2}+a_{n}^{2}-l_{0}}\right] A_{3}^{\prime}(m, n, b, t)(21) \\
u_{z}=\frac{\alpha k}{c^{2}} \sum_{m, n=1}^{\infty}\left[\frac{P_{m}(x)}{\lambda_{m}}\right]\left[\frac{\left(k_{3}+k_{4}\right) \sin 2 a_{n} b}{\lambda_{n}}\right]\left[\frac{(1+v) a_{n}^{2}}{a_{m}^{2}+a_{n}^{2}-1 / c^{2}}\right] \\
\times\left[\varphi_{1}(y) \tau_{1}(t)-\varphi_{2}(y) \tau_{2}(t)\right] \\
+\frac{2 \alpha k \pi}{\xi^{2}} \sum_{m, n, \varsigma=1}^{\infty}\left[\frac{P_{m}(x)}{\lambda_{m}}\right]\left[\frac{\left(k_{3}+k_{4}\right) \sin 2 a_{n} b}{\lambda_{n}}\right]\left[\frac{\varsigma}{\cos \varsigma \pi}\right] \\
{\left[\frac{(1+v) a_{n}^{2}}{a_{m}^{2}+a_{n}^{2}+(\varsigma \pi / \xi)^{2}}\right]\left[\frac{\psi_{1}(y) \tau_{3}(t)-\psi_{2}(y) \tau_{4}(t)}{1+(c \varsigma \pi / \xi)^{2}}\right]} \\
-\alpha \sum_{m, n=1}^{\infty}\left[\frac{P_{m}(x)}{\lambda_{m}}\right]\left[\frac{\left(k_{3}+k_{4}\right) \sin 2 a_{n} b}{\lambda_{n}}\right]\left[\frac{(1+v) a_{n}^{2}}{a_{m}^{2}+a_{n}^{2}-l_{0}}\right] A_{3}(m, n, y, t)
\end{gathered}
$$

Where $\varphi^{\prime}{ }_{1}(b)=\frac{\cosh (b / c)-\sinh (b / c)-1}{1 / c \sinh (\xi / c)}$

$\varphi^{\prime}{ }_{2}(b)=\frac{\cosh ((b-\xi) / c)-\sinh ((b-\xi) / c)-\cosh (b / c)-\sinh (b / c)}{(1 / c) \sinh (\xi / c)}$

$\psi_{1}^{\prime}(b)=\frac{-\cos (\varsigma \pi / \xi) b-(c \varsigma \pi / \xi) \sin (\varsigma \pi / \xi) b+1}{(\varsigma \pi / \xi)}$

$\psi_{2}^{\prime}(b)$

$=\frac{-\cos (\varsigma \pi / \xi)(b-\xi)-(c \varsigma \pi / \xi) \sin (\varsigma \pi / \xi)(b-\xi)+\cos \varsigma \pi}{(\varsigma \pi / \xi)}$

$A^{\prime}{ }_{3}(m, n, h, t)=\int_{0}^{h} A_{3}(m, n, z, t) d z$

\subsection{Determination Of Stress Function:}

Substituting values of (19) in equations (14) to (16) one obtains

$\sigma_{x x}$

$$
\begin{aligned}
& =\frac{\alpha E k}{c^{2}} \sum_{m, n,=1}^{\infty}\left[\frac{P_{m}(x)}{\lambda_{m}}\right]\left[\frac{P_{n}(z)}{\lambda_{n}}\right]\left[\frac{-a_{n}^{2}+1 / c^{2}}{a_{m}^{2}+a_{n}^{2}-1 / c^{2}}\right]\left[\varphi_{1}(y) \tau_{1}(t)\right. \\
& \left.-\varphi_{2}(y) \tau_{2}(t)\right] \\
& \quad+\frac{2 \alpha E k \pi}{\xi^{2}} \sum_{m, n, \zeta=1}^{\infty}\left[\frac{P_{m}(x)}{\lambda_{m}}\right]\left[\frac{P_{n}(z)}{\lambda_{n}}\right]\left[\frac{\varsigma}{\cos (\varsigma \pi)}\right] \\
& {\left[\frac{-a_{n}^{2}-(\varsigma \pi / \xi)^{2}}{a_{m}^{2}+a_{n}^{2}+(\varsigma \pi / \xi)^{2}}\right]}
\end{aligned}
$$

$$
\times \frac{\left[\psi_{1}(y) \tau_{3}(t)-\psi_{2}(y) \tau_{4}(t)\right]}{\left[1+(c \varsigma \pi / \xi)^{2}\right]}
$$

$-\alpha E \sum_{m, n=1}^{\infty}\left[\frac{P_{m}(x)}{\lambda_{m}}\right]\left[\frac{P_{n}(z)}{\lambda_{n}}\right]\left[\frac{a_{m}^{2}-\left(\frac{k_{0}^{2} \pi^{2}}{\xi^{2}}\right)}{a_{m}^{2}+a_{n}^{2}-l_{0}}\right] A_{3}(m, n, y, t)$

Published By:

Blue Eyes Intelligence Engineering 


$$
\begin{aligned}
& \sigma_{y y}= \\
& \begin{aligned}
\left(\frac{\alpha E k}{c^{2}}\right) \sum_{m, n=1}^{\infty}\left[\frac{P_{m}(x)}{\lambda_{m}}\right]\left[\frac{P_{n}(z)}{\lambda_{n}}\right]\left[\frac{-a_{m}^{2}-a_{n}^{2}}{a_{m}^{2}+a_{n}^{2}-1 / c^{2}}\right]\left[\varphi_{1}(y) \tau_{1}(t)-\right. \\
\left.\varphi_{2}(y) \tau_{2}(t)\right] \\
+\frac{2 \alpha E k \pi}{\xi^{2}} \sum_{m, n, \zeta=1}^{\infty}\left[\frac{P_{m}(x)}{\lambda_{m}}\right]\left[\frac{P_{n}(z)}{\lambda_{n}}\right]\left[\frac{\varsigma}{\cos (\varsigma \pi)}\right]\left[\frac{-a_{m}^{2}-a_{n}^{2}}{a_{m}^{2}+a_{n}^{2}+(\varsigma \pi / \xi)^{2}}\right] \\
\quad \times\left[\frac{\psi_{1}(y) \tau_{3}(t)-\psi_{2}(y) \tau_{4}(t)}{\left[1+(c \varsigma \pi / \xi)^{2}\right]}\right] \\
\quad-\alpha E \\
\sum_{m, n=1}^{\infty}\left[\frac{P_{m}(x)}{\lambda_{m}}\right]\left[\frac{P_{n}(z)}{\lambda_{n}}\right]\left[\frac{-a_{m}^{2}-a_{n}^{2}}{a_{m}^{2}+a_{n}^{2}-l_{0}}\right] A_{3}(m, n, y, t)
\end{aligned}
\end{aligned}
$$

$$
\begin{gathered}
\sigma_{z z}=\left(\frac{\alpha E k}{c^{2}}\right) \sum_{m, n=1}^{\infty}\left[\frac{P_{m}(x)}{\lambda_{m}}\right]\left[\frac{P_{n}(z)}{\lambda_{n}}\right]\left[\frac{\frac{1}{c^{2}}-a_{m}^{2}}{a_{m}^{2}+a_{n}^{2}-\frac{1}{c^{2}}}\right] \\
{\left[\phi_{1}(y) \tau_{1}(t)-\phi_{2}(y) \tau_{2}(t)\right]} \\
+\frac{2 \alpha E k \pi}{\xi^{2}} \sum_{m, n, \zeta=1}^{\infty}\left[\frac{P_{m}(x)}{\lambda_{m}}\right]\left[\frac{P_{n}(z)}{\lambda_{n}}\right]\left[\frac{\varsigma}{\cos (\varsigma \pi)}\right]\left[\frac{-(\varsigma \pi / \xi)^{2}-a_{m}^{2}}{a_{m}^{2}+a_{n}^{2}+(\varsigma \pi / \xi)^{2}}\right] \\
\times \frac{\left[\psi_{1}(y) \tau_{3}(t)-\psi_{2}(y) \tau_{4}(t)\right]}{\left[1+(c \varsigma \pi / \xi)^{2}\right]} \\
-\alpha E \\
\sum_{m, n,=1}^{\infty}\left[\frac{P_{m}(x)}{\lambda_{m}}\right]\left[\frac{P_{n}(z)}{\lambda_{n}}\right]\left[\frac{l_{0}-a_{m}^{2}}{a_{m}^{2}+a_{n}^{2}-l_{0}}\right] A_{3}(m, n, y, t)
\end{gathered}
$$

\section{Special Case and Numerical Results}

$$
\begin{aligned}
& \text { Set } f(x, z, t)=\left(1-e^{-t}\right)(x+a)^{2}(x-a)^{2}(z+ \\
& h)^{2}(z-h)^{2} e^{\xi}, \\
& g(x, z, t)=\left(1-e^{-t}\right)(x+a)^{2}(x-a)^{2}(z+h)^{2}(z-
\end{aligned}
$$

$h)^{2}$,

$$
\delta=\frac{8\left(k_{1}+k_{2}\right) k \pi}{h^{2}}, \mathrm{a}=4 \mathrm{~m}, \mathrm{~b}=5 \mathrm{~m}, \mathrm{~h}=0.2 \mathrm{~m}, \xi=1.5 \mathrm{~m}, \mathrm{k}=
$$

0.86 , heating time $=5$ minutes in the equation (17) to obtain

$$
\begin{aligned}
& \frac{T(x, y, z, t)}{\delta}=\sum_{m=1}^{\infty} \sum_{n=1}^{\infty} \sum_{\eta=1}^{\infty}(-1)^{(\eta+1 / 2)}(\eta+ \\
& \left.\frac{1}{2}\right)\left(\frac{P_{n}(x)}{\mu_{n}}\right)\left(\frac{P_{m}(z)}{\lambda_{m}}\right)\left(\frac{1}{1-q^{2}}\right) \\
& \times\left[\frac{a_{n} \cos ^{2}\left(a_{n}\right)-\cos \left(a_{n}\right) \sin \left(a_{n}\right)}{a_{n}{ }^{2}}\right] \times[\Phi(y) e-
\end{aligned}
$$

$\Psi(y)]$

$$
\times \int_{0}^{t}\left(1-e^{-t^{\prime}}\right) e^{-0.86\left(q^{2}+\left(\eta+\frac{1}{2}\right)^{2} \pi^{2}\right)\left(t-t^{\prime}\right)} d t^{\prime}
$$

\section{Material Properties}

The numerical calculation has been carried out for a rectangular plate made of pure Aluminium material with following material properties and dimensions.

Young's modulus of elasticity $=\mathrm{E}=70 \times 10^{9} \mathrm{Nm}^{-2}$

Poisson ratio $=\mathrm{v}=0.281$

Density $=\rho=2700 \mathrm{Kgm}^{-3}$

Specific heat at constant pressure $=\mathrm{Cp}=921 \mathrm{JKg}^{-1} \mathrm{~K}^{-1}$

Thermal conductivity $=237 \mathrm{Wm}^{-1} \mathrm{~K}^{-1}$ $\mathrm{K}^{-1}$

Linear coefficient of thermal expansion $=\alpha=2.3 \times 10^{-5}$

Thermal Diffusivity $=\mathrm{k}=95 \times 10^{-6} \mathrm{~m}^{2} \mathrm{sec}^{-1}$

Dimensions

Length of rectangular plate $(\mathrm{x}$-direction $)=4 \mathrm{~m}$

Breadth of rectangular Plate $(\mathrm{y}$-direction $)=5 \mathrm{~m}$

Thickness of rectangular plate $(\mathrm{z}$-direction $)=0.2 \mathrm{~m}$

\section{RESULTS AND DISCUSSION}

In this article an inverse thermoelastic problem of a rectangular plate is discussed and expression for unknown surface temperature is determined on the boundary $y=b$, along the y-axis, when the temperature at some internal point is known and both the boundaries along $\mathrm{x}$ and $\mathrm{z}$-axis are maintained at zero temperature. A mathematical model is developed for the function $f(x, z, t)=\left(1-e^{-t}\right)(x+$ $a)^{2}(x-a)^{2}(z+h)^{2}(z-h)^{2} e^{\xi} \quad$ and displacement components, and stress components are computed. As an example, numerical calculations have been done for a rectangular plate made up of Aluminium metal and the results are graphically represented.

From Fig. 1 and Fig.3 the displacement functions Ux and Uz show a compressive trend in the mid portion of the rectangular beam along Y-direction, while from Fig.2 it is observed that the displacement function Uy shows an elongation along the same direction. Fig.4 and Fig. 6 indicate that the stress functions $\sigma_{\mathrm{xx}}$ and $\sigma_{\mathrm{zz}}$ induces a stress of compressive nature along the length of the rectangular beam in the Y-direction, while, fig. 5 indicates that the stress component $\sigma_{y y}$ induces a tensile stress along the same direction ie along the direction of existence of temperature gradient.

Thus, it is observed that displacement and stress along the direction of temperature gradient, here Y-direction, produces a tensile stress. Displacement and stresses along $\mathrm{X}$ and Z-directions may be compressive, due to lack of temperature gradient. The possible cause of thermal stressstrain in solid bodies can be attributed to the existence of temperature gradient and the resulting heat flux. 


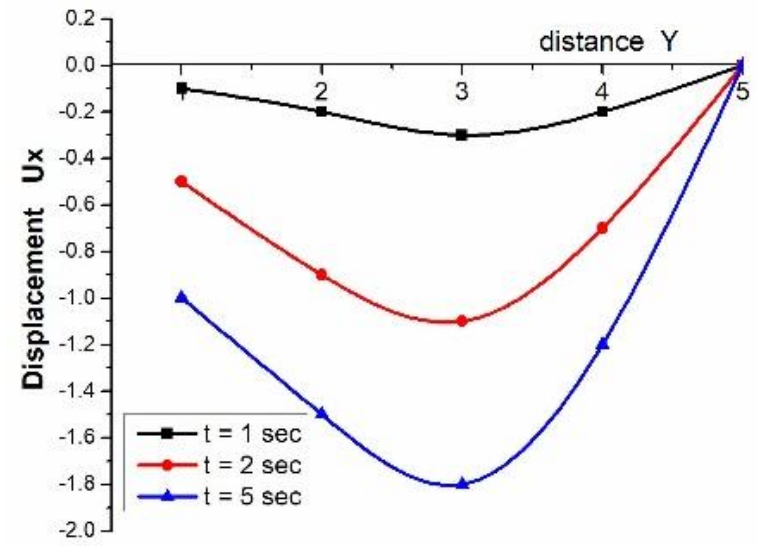

Fig 1. Variation of displacement $\mathrm{Ux}$ with distance $\mathrm{y}$.

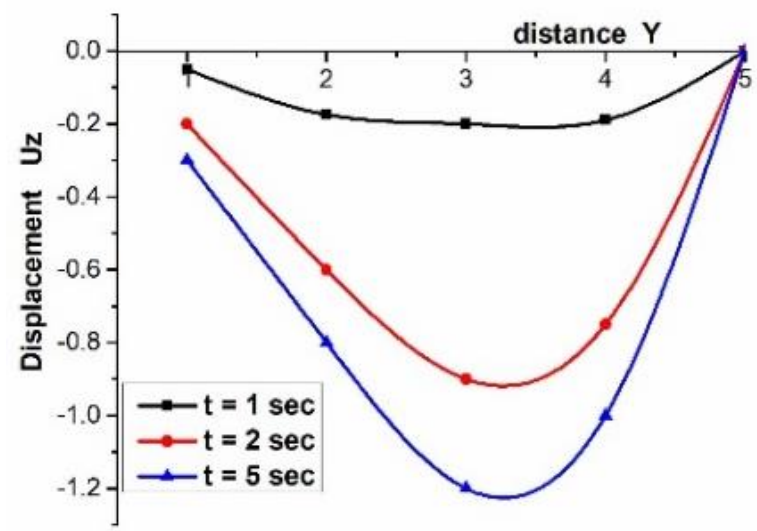

Fig 3. Variation of displacement $\mathrm{Uz}$ with distance $\mathrm{y}$.

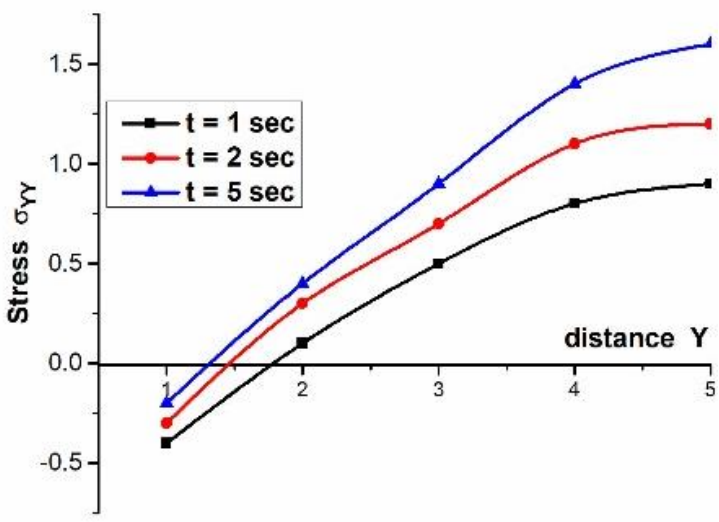

Fig 5. Variation of stress $\sigma_{y y}$ with distance $y$.

\section{CONCLUSION}

In this article an inverse thermoelastic problem of a rectangular plate is discussed and unknown surface temperature is determined on the boundary $y=b$, along the $y$ axis, when the temperature at some internal point is known and both the boundaries along $\mathrm{x}$ and $\mathrm{z}$-axis are maintained at zero temperature. Finite Marchi-Fasulo transform and Laplace transform techniques have been effectively used to obtain expression for temperature, displacement and stress. The results are obtained in the form of infinite series. Any particular case of special interest can be derived by assigning suitable values to the parameters and functions in
Published By:

Blue Eyes Intelligence Engineering and Sciences Publication

(C) Copyright: All rights reserved.

Fig 6. Variation of stress $\sigma_{\mathrm{zz}}$ with distance $y$.

the expressions from (20) to (25). This method of thermoelasticity study is effective as compared to other analytical-numerical methods and results obtained show a good agreement with earlier studies.The study can be helpful in various design and structural engineering processes, particularly, the strain and stresses in rectangular shaped base or foundations of furnaces, containers, and nuclear reactors can be evaluated.

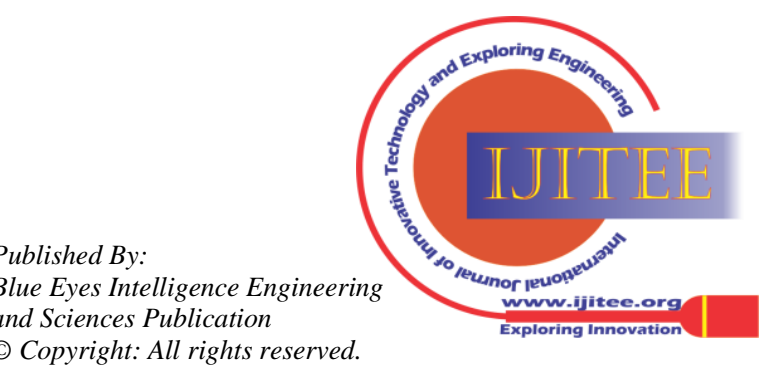




\section{REFERENCE}

1. Ahire, Y. M., Hamoud, A. A., Ghadle, K. P., \& Sable, N. P. (2020) Thermal stresses and temperature profile in functionally graded material with internal heat generation in rectangular plate. International Journal of Advanced Science and Technology, 29(4), 2137-2147.

http://sersc.org/journals/index.php/IJAST/article/view/10494

2. Amiri Delouei, A., Emamian, A., Karimnejad, S., \& Sajjadi, H. (2019). A closed-form solution for axisymmetric conduction in a finite functionally graded cylinder. International Communications in Heat and Mass Transfer, 108, 104280. https://doi.org/10.1016/j.icheatmasstransfer.2019.104280

3. Amiri Delouei, A., Emamian, A., Karimnejad, S., Sajjadi, H., \& Tarokh, A. (2019). On 2D asymmetric heat conduction in functionally graded cylindrical segments: A general exact solution. International Journal of Heat and Mass Transfer, 143, 118515. https://doi.org/10.1016/j.ijheatmasstransfer.2019.118515

4. Arslantürk, C. (2018). Variation of parameters method for optimizing annular fins with variable thermal properties. Pamukkale University Journal of Engineering Sciences, 24(1), 1-7. https://doi.org/10.5505/pajes.2017.78736

5. Chaudhari, K. K., \& Sutar, C. S. (2019). Thermoelastic response of a rectangular plate under hyperbolic heat conduction model in differential transform domain. International Journal of Engineering and Advanced Technology, 9(1), 692-699. https://doi.org/10.35940/ijeat.F9047.109119

6. Chen, P. Y. P. (1988). TRANSIENT THERMAL STRESSES IN A RECTANGULAR PLATE DUE TO NONUNIFORM HEAT TRANSFER COEFFICIENTS. Journal of Thermal Stresses, 11(2), 115-125. https://doi.org/10.1080/01495738808961924

7. Delouei, A. A., Emamian, A., Karimnejad, S., Sajjadi, H., \& Jing, D. (2020). Asymmetric Conduction in an Infinite Functionally Graded Cylinder: Two-Dimensional Exact Analytical Solution Under General Boundary Conditions. Journal of Heat Transfer, 142(4), 044505. https://doi.org/10.1115/1.4046306

8. Grysa, K., \& Kozłowski, Z. (1983). One-dimensional problems of temperature and heat flux determination at the surfaces of a thermoelastic slab. Nuclear Engineering and Design, 74(1), 1-14. https://doi.org/10.1016/0029-5493(83)90135-8

9. Güngör, O., \& Arslantürk, C. (2019). Variation of parameters method for a three-dimensional problem of condensation film on an inclined rotating disk. Journal of Applied Mathematics and Computational Mechanics, 18(1), 15-28. https://doi.org/10.17512/jamcm.2019.1.02

10. Kaur, I., Lata, P. (2019). Transversely isotropic thermoelastic thin circular plate with constant and periodically varying load and heat source. International Journal of Mechanical and Materials Engineering, 14(10), 10. https://doi.org/10.1186/s40712-019-0107-4

11. Kaur,I., Lata, P. (2019). Thermomechanical interactions in transversely isotropic thick circular plate with axisymmetric heat supply. Structural Engineering and Mechanics, 69(6), 607-614. https://doi.org/https://doi.org/10.12989/sem.2019.69.6.607

12. Manthena, V., Lamba, N., Kedar, G. (2018). Thermoelastic Analysis of a Rectangular Plate with Nonhomogeneous Material Properties and Internal Heat Source. Journal of Solid Mechanics, 10(1), 200 215. http://jsm.iau-arak.ac.ir/article_539719.html

13. Marin, M., \& Craciun, E. M. (2017). Uniqueness results for a boundary value problem in dipolar thermoelasticity to model composite materials. Composites Part B: Engineering, 126, 27-37. https://doi.org/10.1016/j.compositesb.2017.05.063

14. Marin, Marin, \& Öchsner, A. (2017). The effect of a dipolar structure on the Hölder stability in Green-Naghdi thermoelasticity. Continuum Mechanics and Thermodynamics, 29(6), 1365-1374. https://doi.org/10.1007/s00161-017-0585-7

15. Noda, N., Ashida, F., \& Tsuji, T. (1989). An Inverse Transient Thermoelastic Problem for a Transversely-Isotropic Body. Journal of Applied Mechanics, 56(4), 791-797. https://doi.org/10.1115/1.3176173

16. Noda, N., Hetnarski, R. B., \& Tanigawa, Y. (2003). Thermal Stresses (N. Noda, R. B. Hetnarski, \& Y. Tanigawa (eds.); 2nd ed.) CRC Press. https://doi.org/10.1201/9780203735831

17. Robert J. Adams, Bret,C. W. (1999). THERMOELASTIC VIBRATIONS OF A LAMINATED RECTANGULAR PLATE SUBJECTED TO A THERMAL SHOCK. Journal of Thermal Stresses, 22(9), 875-895. https://doi.org/10.1080/014957399280607

18. Roy H., Bagade, S. H.. Khobragade, N. W. (2013). Thermal Stresses of a Semi infinite Rectangular Beam. Int. J. of Engg. And Innovative Technology, 3(1), 442-445. https://www.ijeit.com/Vol 3/Issue 1/IJEIT1412201307_82.pdf
19. Tanigawa, Y., Ootao, Y. (1999). THREE-DIMENSIONAL TRANSIENT THERMAL STRESSES OF FUNCTIONALLY GRADED RECTANGULAR PLATE DUE TO PARTIAL HEATING. Journal of Thermal Stresses, 22(1), 35-55. https://doi.org/10.1080/014957399281048

20. Xue, T., Zhang, X., \& Tamma, K. K. (2019). A non-local dissipative Lagrangian modelling for generalized thermoelasticity in solids. Applied Mathematical Modelling, 73, 247-265. https://doi.org/10.1016/j.apm.2019.04.004

21. Youssef, H. M., Oreijah, M., \& Alsharif, H. S. (2019). ThreeDimensional Generalized Thermoelasticity with Variable Thermal Conductivity. International Journal of Mathematical and Computational Sciences, 13(5), 108-116 https://doi.org/10.5281/zenodo.2702917

\section{AUTHORS PROFILE}

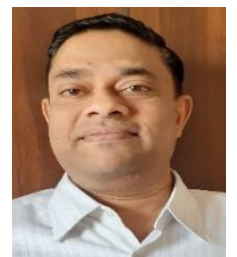

Dr. Sanjay $\mathbf{H}$ Bagade, is currently working as Head and Associate Professor in Department of Physics, Bajaj College of Science, Wardha (MS). He has about 20 years of teaching experience and has to his credit several research articles published in national and international journals. He also has published three books on thermoelasticity. His area of research Mathematical Physics. 